\title{
LEVANTAMENTO SOBRE DIRETIVAS DA W3C PARA A PROMOÇÃO DA ACESSIBILIDADE À DEFICIENTES AUDITIVOS NA WEB
}

\author{
W3C Guidelines to Promote Hearing Impaired Web Accessibility: A \\ Survey
}

\author{
Vanessa dos Anjos Borges ${ }^{1}$ \\ ${ }^{1}$ Centro Estadual de Educação Tecnológica Paula Souza \\ Faculdade de Tecnologia de Presidente Prudente \\ Rua Terezina, 75, Vila Paulo Roberto, Presidente Prudente/SP-Brasil \\ vanessa.borges2@fatec.sp.gov.br
}

\begin{abstract}
RESUMO - A inclusão de pessoas com deficiência em diferentes meios sociais vem se tornando objeto de estudo de pesquisas. Isso se dá ao fato do crescente número de pessoas com deficiência, que possuem por direto ter acesso a todos os recursos e informações disponíveis em todos os meios de comunicação. A partir de uma pesquisa descritiva, neste artigo foram identificados critérios de sucesso contidos no Guia de Acessibilidade para o Conteúdo Web (Web Content Accessibility Guidelines) definida pela W3C (World Wide Web Consortium) voltados para a promoção da inclusão dos deficientes auditivos na Web, auxiliando futuros trabalhos na área a fim de garantir o desenvolvimento de aplicações acessíveis para deficientes auditivos. Palavras-chave: inclusão; internet; legislação brasileira.
\end{abstract}

Recebido em: 29/05/2018

Revisado em: 21/08/2018

Aprovado em: 28/08/2018
ABSTRACT - The inclusion of people with disabilities in different social environments has become the object of research studies. This is due to the increasing number of people with disabilities, who have right access to all resources and information available in all media. From a descriptive research, in this article were identified success criteria contained in the Web Content Accessibility Guide (WCAG) defined by the W3C (World Wide Web Consortium) aimed at promoting the inclusion of the hearing impaired on the Web, helping future work in the area to ensure the development of accessible applications for the hearing impaired.

Keywords: inclusion; internet; brazilian legislation. 


\section{INTRODUÇÃO}

Para promoção da inclusão de pessoas com deficiência ( $P C D)$, a Lei de Cotas de 1991 regulamenta a contratação de pessoas com deficiências, esclarecendo que empresas que possuam mais do que cem funcionários têm o dever de a preencher de dois a cinco por cento dos seus cargos com beneficiários reabilitados, ou pessoas com deficiência. Essa variação de porcentagem depende da quantidade de colaboradores da organização, e essa é estabelecida pela lei (BRASIL, 1991).

Para a inserção de pessoas com deficiência tanto no mercado de trabalho quanto em outros meios de socialização, é necessário realizar adequações para fornecer um ambiente favorável para a inclusão desses indivíduos (KONZA, 2008; SACHS; SCHREUER, 2011; SIMPLICAN et al., 2015).

A Internet é o canal de comunicação mais crescente dos últimos tempos, e presente em todas as organizações sociais. De acordo com o Instituto Brasileiro de Geografia e Estatística (IBGE, 2017a) cerca de 86,7 milhões de pessoas com 10 anos ou mais de idade acessaram a Internet no Brasil em 2013.

Ainda segundo o Censo Demográfico Brasileiro de 2010 (IBGE, 2017b), cerca de 43 milhões de pessoas possuem algum tipo de deficiência visual, auditiva ou motora. Desse total, 9,7 milhões de pessoas declararam possuir deficiência auditiva. Os valores relacionados ao número de deficientes levantados nesse censo foram questionados por diversos pesquisadores pela sua abordagem metodológica na caracterização de pessoas com deficiência (SILVA, 2013).

Porém, mesmo com as inconsistências identificadas no censo, é notável que essa parcela da população que possui algum tipo de deficiência possui a necessidade de ser incluída no contexto social. As características humanas influenciam diretamente a participação das pessoas em sua relação com sistemas interativos. Sistemas interativos que lidam com informações requerem capacidades cognitivas para processar as informações e aprender a utilizá-los (BARBOSA; SILVA, 2010).

A W3C (World Wide Web Consortium) é umas das organizações responsáveis pela padronização da World Wide Web. Para a garantia da acessibilidade das pessoas com deficiência, a W3C gerenciou a iniciativa Web Accessibility Initiative (WAI) (W3C, 2017a), que teve por finalidade a elaboração de guias para a promoção da acessibilidade. A partir da WAl foram elaborados os seguintes guias: Guia de Acessibilidade para o Conteúdo Web (Web Content Accessibility Guidelines WCAG), o Guia de Acessibilidade para Agentes do Usuário (User Agent Accessibility Guidelines - UAAG) e o Guia de Acessibilidade para Ferramentas de Autoria (Authoring Tool Accessibility Guidelines ATAG).

O Guia de Acessibilidade para o Conteúdo Web (Web Content Accessibility Guidelines - WCAG) (W3C, 2017b) descreve um conjunto de recomendações para promoção da acessibilidade na Web para pessoas com incapacidades, como cegueira e baixa visão, surdez e baixa audição, dificuldades de aprendizagem, limitações cognitivas, limitações de movimentos, incapacidade de fala e fotossensibilidade. 0 foco das diretivas contidas no WCAG é tornar as páginas Web acessíveis a todos os usuários da Internet em geral.

A Legislação Brasileira vem contribuindo significativamente com 0 estabelecimento de normas voltadas para a acessibilidade de pessoas com deficiência. Recentemente, o Marco Civil da Internet (Lei n. 12.965, de 23 de Abril de 2014) (BRASIL, 2014) foi considerado também um grande avanço na Legislação Brasileira em relação às regulamentações de uso da Internet no Brasil. Essa lei estabelece princípios, garantias, direitos e deveres para o uso da Internet no Brasil. Em seu artigo $4^{\circ}$, a lei estabelece a obrigatoriedade da adesão a padrões tecnológicos que permitam a comunicação, a acessibilidade e a interoperabilidade na Web. 
Anteriormente a essa lei, outros decretos e leis já estabeleciam normas para garantia dos direitos de pessoas com deficiência. A Convenção dos Direitos das Pessoas com Deficiência, instituída em 2007 pela Assembleia Geral das Nações Unidas foi um marco para garantir o direito de inclusão das pessoas com deficiência. A Lei n. 13.146, de 6 de Julho de 2015 baseada na Convenção Internacional sobre os Direitos das Pessoas com Deficiência, estabelece o exercício dos direitos e das liberdades fundamentais das pessoas com deficiência, visando à sua inclusão social e cidadania (BRASIL, 2015).

A respeito das pessoas com deficiência auditiva, são necessárias adaptações principalmente quanto à comunicação, entre os próprios colaboradores, ou a mudança dos meios de comunicação, como telefones apropriados, ou até mesmo softwares com suporte e tradução a língua dos deficientes auditivos.

Entre os trabalhos relacionados a essa temática, no artigo desenvolvido pelos autores Flor, Vanzin e Ulbricht (2013) é apresentada uma discussão a respeito dos padrões de acessibilidade na web para surdos, onde foram identificados critérios de sucesso da WCAG voltados para apoiar questões relacionadas à linguagem na surdez, não incluindo outras recomendações relacionadas à deficiência auditiva como a baixa audição.

Nesse sentido, o propósito desse artigo amplia a ideia proposta pelos autores, apresentando as diretivas contidas no Guia de Acessibilidade para o Conteúdo Web (WCAG) no que tange à promoção da acessibilidade aos indivíduos surdos ou de baixa audição na Web.

\section{PROCEDIMENTOS METODOLÓGICOS}

Esse trabalho caracteriza-se como uma pesquisa descritiva. As pesquisas descritivas "vão além da simples identificação da existência de relações entre variáveis, e pretendem determinar a natureza dessa relação" (GIL, 2010, p.42).

Levando em consideração o modelo WCAG proposto pela W3C, foi realizada uma análise dos critérios de sucesso para atender as necessidades dos deficientes auditivos.

Dentre as principais necessidades identificadas de acordo com a análise exploratória realizada sobre o tema anteriormente pelo autor, foram levadas em consideração as necessidades de tradução de áudio, interpretação de dados em línguas de sinais (para deficientes auditivos que não são bilíngues, ou seja, não possui a capacidade de interpretar a língua portuguesa), e nitidez de som disponível (para pessoas com baixa audição) para que as interfaces Web sejam acessíveis os deficientes auditivos (FLOR; VANZIN; ULBRICHT, 2013; SKLIAR, 2012).

\section{RESULTADOS E DISCUSSÃO}

De acordo com as necessidades identificadas, dos 61 critérios de sucesso contidos no WCAG, 10 foram selecionados para atendê-las. O Quadro 1 relaciona os critérios de sucesso voltados a suprimir as necessidades para promover a acessibilidade aos deficientes auditivos. Tais necessidades estão relacionadas a necessidade de transcrição de áudio devido a perda do sentido auditivo. 
Quadro 1. Critérios de Sucesso segundo o WCAG 2.0 para Pessoas com Deficiência Auditiva.

\begin{tabular}{|c|c|}
\hline Número & Descrição \\
\hline 1.2 .1 & $\begin{array}{l}\text { O conteúdo das mídias de áudio pré-gravado devem possuir um meio alternativo de } \\
\text { compreensão }\end{array}$ \\
\hline 1.2 .2 & $\begin{array}{l}\text { Legendas pré-gravadas são fornecidas para áudio pré-gravado presente em um } \\
\text { conteúdo multimídia, exceto quando o conteúdo já possui algum meio alternativo } \\
\text { de compreensão }\end{array}$ \\
\hline 1.2 .4 & Legendas ao vivo são fornecidas para todo o conteúdo de áudio ao vivo transmitido \\
\hline 1.2 .6 & A interpretação do conteúdo é realizada por meio de Língua de Sinais pré-gravadas \\
\hline 1.2 .8 & $\begin{array}{l}\text { É fornecida uma versão alternativa do conteúdo utilizando multimídia pré-gravada } \\
\text { (apresentação de vídeos) }\end{array}$ \\
\hline 1.4 .2 & $\begin{array}{l}\text { Se um som numa página Web tocar automaticamente durante mais de } 3 \text { segundos, } \\
\text { o usuário deve poder controlar o som, como pausá-lo, pará-lo, ou controlar o } \\
\text { volume de forma independente }\end{array}$ \\
\hline 1.4 .7 & $\begin{array}{l}\text { Conteúdos de áudio que possuam essencialmente fala em primeiro plano (exceto } \\
\text { CAPTCHA de áudio, logotipo de áudio, e áudio com vocalização musical), o áudio não } \\
\text { deve conter sons de fundo, ou os sons de fundo podem ser desligados, ou os sons de } \\
\text { fundo possuem no mínimo, } 20 \text { decibéis a menos do que o conteúdo da fala em } \\
\text { primeiro plano }\end{array}$ \\
\hline 3.1 .3 & O idioma da página Web deve ser definido programaticamente \\
\hline 3.1 .4 & $\begin{array}{l}\text { O idioma da página Web deve ser definido programaticamente exceto para os } \\
\text { nomes próprios, os termos técnicos, palavras escritas num idioma indeterminado e } \\
\text { palavras ou frases que se tenham convertido em linguagem corrente da língua } \\
\text { utilizada no texto que as envolve }\end{array}$ \\
\hline 4.1 .1 & $\begin{array}{l}\text { Os elementos que compões uma página Web (tags) devem ser bem definidas para } \\
\text { permitir interpretação automática }\end{array}$ \\
\hline
\end{tabular}

A primeira necessidade de tradução de áudio, está relacionada a geração de conteúdo alternativo relacionado a áudio pré-gravado. O critério de sucesso 1.2.1 - O conteúdo das mídias de áudio pré-gravado devem possuir um meio alternativo de compreensão. Em sua definição completa, ela preconiza alternativas de interpretação tanto para áudio quanto vídeos pré gravados. No que tange os deficientes auditivos, um exemplo de alternativa para áudios prégravados é uma descrição textual em português ou icônica (por exemplo, em SignWriting, uma transcrição em ícones de línguas de sinais (STUMPF, 2005)) alternativa contida em um link ou em uma seção da página Web.

Legendas, tanto em vídeo ao vivo ou pré-gravados são alternativas para a necessidade de tradução de áudio. Os critérios de sucesso 1.2.2 - Legendas prégravadas são fornecidas para áudio pré- gravado presente em um conteúdo multimídia, exceto quando o conteúdo já possui algum meio alternativo de compreensão e 1.2.4 - Legendas ao vivo são fornecidas para todo o conteúdo de áudio ao vivo transmitido.

Uma alternativa válida para tradução de informações de áudio encontra-se no critério de sucesso 1.2.6 - A interpretação do conteúdo é realizada por meio de Língua de Sinais pré-gravadas e 1.2.8 - É fornecida uma versão alternativa do conteúdo utilizando multimídia pré-gravada (apresentação de vídeos).

Um exemplo onde pode-se verificar a aplicação desses dois critérios de sucesso estão no projeto LE@D - Laboratório de Educação a Distância para Pessoas com Deficiência (DELL, 2017) criado pela Dell, em parceria com a Universidade Estadual do Ceará, onde foram desenvolvidas uma aplicação Web e outro aplicativo para a 
plataforma Android que provêm o ensino a distância, aumentando as oportunidades de empregabilidade de pessoas com deficiência no Brasil.

Nas aplicações são oferecidas alternativas em vídeo para tradução do conteúdo em LIBRAS (Língua Brasileira de Sinais). A Figura 1 apresenta o funcionamento do portal do projeto LE@D com a transcrição do conteúdo escrito em vídeo.

Em relação a necessidades de pessoas com baixa audição, os critérios de sucesso 1.4.2 - Se um som numa página Web tocar automaticamente durante mais de 3 segundos, o usuário deve poder controlar $o$ som, como pausá-lo, pará-lo, ou controlar o volume de forma independente e 1.4.7 Conteúdos de áudio que possuam essencialmente fala em primeiro plano (exceto CAPTCHA de áudio, logotipo de áudio, $e$ áudio com vocalização musical), o áudio não deve conter sons de fundo, ou os sons de fundo podem ser desligados, ou os sons de fundo possuem no mínimo, 20 decibéis a menos do que o conteúdo da fala em primeiro plano trazem requisitos necessários para a compreensão do conteúdo disponibilizado por áudio.

Figura 1. Tradução do conteúdo em Português do Brasil para LIBRAS: portal do projeto LE@D.

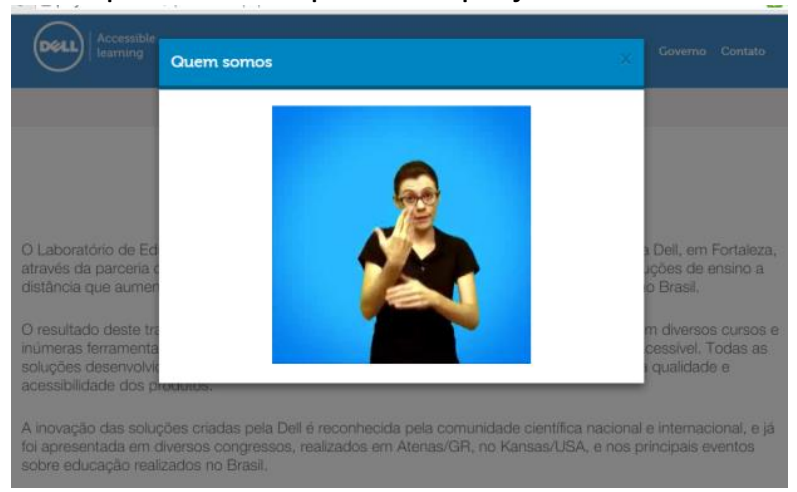

Fonte: Dell (2017).

O volume do áudio nas páginas Web deve possuir regulagens para capacitar 0 aumento dos decibéis, também devem prover a capacidade de pausa e continuidade do áudio, propiciando ao usuário retomar um trecho de áudio para nova escuta e compreensão. A nitidez do áudio também é de suma importância, uma vez que a interferência no áudio pode corromper a mensagem a ser transmitida.

O idioma exibido na página Web também é importante para propiciar o entendimento do conteúdo por deficientes auditivos. Os critérios de sucesso 3.1.3 - O idioma da página Web deve ser definido programaticamente e 3.1.4 - O idioma da página Web deve ser definido programaticamente exceto para os nomes próprios, os termos técnicos, palavras escritas num idioma indeterminado e palavras ou frases que se tenham convertido em linguagem corrente da língua utilizada no texto que as envolve estão relacionados à necessidade de disponibilizar o conteúdo em diversas línguas.

A tradução de um conteúdo em Língua Portuguesa do Brasil para a transcrição de Línguas de Sinais em SignWriting é um exemplo de aplicação desse critério de sucesso. Em caso de nomes próprios, termos técnicos e jargões devem ser preservados e informações complementares serem apresentadas para a compreensão do contexto.

Durante a realização da análise e da identificação dos critérios de sucesso, um critério se destacou entre os demais, definindo uma nova necessidade ainda não identificada.

Uma parcela significativa dos deficientes auditivos não completa todo o ciclo de ensino, devido à diversos fatores econômicos e sociais, e principalmente pela falta de estrutura para desenvolverem seus estudos. Grande parte desses indivíduos abandonam por não possuírem o ambiente ideal para sua formação (ROCHA; LUIZ; ZULIAN, 2003). Portanto, também foi elegido como critério de sucesso para acessibilidade de deficientes auditivos na Web o critério de sucesso 3.1.5 - Quando o texto exigir uma capacidade de leitura mais avançada do que ensino básico, é necessário disponibilizar um conteúdo suplementar. 


\section{CONSIDERAÇÕES FINAIS}

Este artigo teve como proposta evidenciar os principais critérios de sucesso do WCAG 2.0 para proporcionar acessibilidade aos deficientes auditivos na Web. Assim, a partir da análise das necessidades dos deficientes auditivos foi possível definir os requisitos essenciais que uma página Web deve conter para que elas sejam acessíveis a esses indivíduos.

A principal contribuição desse artigo é orientar desenvolvedor e web designer no que concerne a conceber soluções que atendam às necessidades dos deficientes auditivos

É interessante ressaltar um requisito encontrado a partir da análise do contexto onde o deficiente auditivo é inserido: o critério de sucesso 3.1.5 - Quando o texto exigir uma capacidade de leitura mais avançada do que ensino básico, é necessário disponibilizar um conteúdo suplementar. Apenas 6,66\% das pessoas com deficiência possui ensino superior completo, e $61,13 \%$ não possuem instrução ou fundamental incompleto (ISOCIAL, 2017).

Muitos deles não dão continuidade em sua formação, o que pode ser uma restrição para acessibilidade nas páginas Web, uma vez que a utilização de uma linguagem extremamente formal pode ser uma barreira para o acesso a informação.

\section{REFERÊNCIAS}

BARBOSA, S. D. J.; SILVA, B. S. Interação humano computador. 1. ed. Rio de Janeiro: Campus, 2010.

BRASIL. Lei no 8.213, de 24 de julho de 1991. Dispõe sobre os Planos de Benefícios da Previdência Social e dá outras providências. Diário Oficial da União, 1991.

BRASIL. Lei $n^{\circ}$ 12.965, de 23 de Abril de 2014. Marco Civil da Internet. Diário Oficial da União, 2014.
BRASIL. Lei $n^{\circ}$ 13.146, de 6 de Julho de 2015. Lei Brasileira de Inclusão da Pessoa com Deficiência (Estatuto da Pessoa com Deficiência). Diário Oficial da União, 2015.

DELL. Projeto LE@D. Disponível em: http://projetolead.com.br/index.php. Acesso em: 18 out. 2017.

FLOR, C.S; VANZIN, T.; ULBRICHT, V. Recomendações da WCAG 2.0 (2008) e a acessibilidade de surdos em conteúdos da Web. Revista Brasileira de Educação Especial, v. 19, n. 2, p. 161-168, 2013. https://doi.org/10.1590/S141365382013000200002

GIL, A. C. Como elaborar projetos de pesquisa. São Paulo: Atlas, 2010.

IBGE. Censo Demográfico 2010: características urbanísticas do entorno dos domicílios. Disponível em: $\mathrm{ftp}$ ://ftp.ibge.gov.br/Censos/Censo_Demogra fico_2010/Entorno_dos_Domicilios/xls/Brasil .zip. Acesso em: 18 out. 2017a.

IBGE. Censo Demográfico 2010: características gerais da população, religião e pessoas com deficiência. Disponível em: $\mathrm{ftp}: / / \mathrm{ftp}$. ibge.gov.br/Censos/Censo_Demogra fico_2010/Caracteristicas_Gerais_Religiao_D eficiencia/xls/Brasil_xls.zip. Acesso em: 18 out. 2017b.

ISOCIAL. 0 ingresso da pessoa com deficiência no ensino superior e os avanços para um mercado de trabalho inclusivo. 2017. Disponível em: $<$ http://blog.isocial.com.br/o-ingresso-dapessoa-com-deficiencia-no-ensinosuperior/>. Acesso em: 09 ago. 2018.

KONZA, D. Inclusion of students with disabilities in new times: responding to the challenge. In: KELL, P. et al. (eds). Learning and the learner: exploring learning for new times. University of Wollongong, 2008. 
ROCHA, E. F.; LUIZ, A.; ZULIAN, M. A. Reflexões sobre as possíveis contribuições da terapia ocupacional nos processos de inclusão escolar. Revista de Terapia Ocupacional da Universidade de São Paulo, v.14, n.2, p.72-78, 2003. https://doi.org/10.11606/issn.22386149.v14i2p72-78

SACHS, D.; SCHREUER, N. Inclusion of students with disabilities in higher education: performance and participation in student's experiences. Disability Studies Quarterly, v.31, n.2, 2011. https://doi.org/10.18061/dsq.v31i2.1593

SILVA, R. A. L. Recomendações para acessibilidade aos surdos de auxílio aos designers na criação e na implementação de ambientes web. 2013. Dissertação (Mestrado) - Universidade Federal do Paraná, Curitiba, 2013.

SKLIAR, C. A. (Org.). A surdez: um olhar sobre as diferenças. Porto Alegre: Mediação, 2012.

SIMPLICAN, S. C. et al. Defining social inclusion of people with intellectual and developmental disabilities: An ecological model of social networks and community participation. Research in developmental disabilities, v.38, p.18-29, 2015. https://doi.org/10.1016/i.ridd.2014.10.008

STUMPF, M. R. Aprendizagem de escrita de língua de sinais pelo sistema SignWriting: língua de sinais no papel e no computador. 2005. Tese (Doutorado) - Universidade Federal do Rio Grande do Sul, Porto Alegre, 2005.

W3C. Web Accessibility Initiative. Disponível em: http://www.w3.org/WAl/. Acesso em: 18 out. 2017a.

W3C. Web Content Accessibility Guidelines 2.0. Disponível em: http://www.w3.org/Translations/WCAG20-
pt-T/WCAG20-pt-PT-20141024/\#guidelines>> Acesso em: 18 out. 2017b. 